\title{
Optimal Pattern Synthesis of Linear Antenna Array Using Grey Wolf Optimization Algorithm
}

\author{
Prerna Saxena and Ashwin Kothari \\ Department of Electronics and Communication Engineering, Visvesvaraya National Institute of Technology, Nagpur 440010, India \\ Correspondence should be addressed to Prerna Saxena; prerna.saxena2707@gmail.com
}

Received 3 November 2015; Revised 18 January 2016; Accepted 13 March 2016

Academic Editor: Felipe Cátedra

Copyright ( 2016 P. Saxena and A. Kothari. This is an open access article distributed under the Creative Commons Attribution License, which permits unrestricted use, distribution, and reproduction in any medium, provided the original work is properly cited.

\begin{abstract}
The aim of this paper is to introduce the grey wolf optimization (GWO) algorithm to the electromagnetics and antenna community. GWO is a new nature-inspired metaheuristic algorithm inspired by the social hierarchy and hunting behavior of grey wolves. It has potential to exhibit high performance in solving not only unconstrained but also constrained optimization problems. In this work, GWO has been applied to linear antenna arrays for optimal pattern synthesis in the following ways: by optimizing the antenna positions while assuming uniform excitation and by optimizing the antenna current amplitudes while assuming spacing and phase as that of uniform array. GWO is used to achieve an array pattern with minimum side lobe level (SLL) along with null placement in the specified directions. GWO is also applied for the minimization of the first side lobe nearest to the main beam (near side lobe). Various examples are presented that illustrate the application of GWO for linear array optimization and, subsequently, the results are validated by benchmarking with results obtained using other state-of-the-art nature-inspired evolutionary algorithms. The results suggest that optimization of linear antenna arrays using GWO provides considerable enhancements compared to the uniform array and the synthesis obtained from other optimization techniques.
\end{abstract}

\section{Introduction}

Antenna arrays play a significant role in modern wireless communication systems because of their advantages over single antenna element such as high directivity, high gain, and beam steering capabilities. These characteristics of antenna arrays make them suitable for applications like beam forming and null positioning [1]. Null positioning in order to eliminate the unwanted signals which cause interference is probably one of the most important goals of array synthesis [2, 3]. Moreover, producing a directive beam at a particular direction while keeping the side lobe level (SLL) small to avoid interference with other radiating sources is another important aim of array synthesis.

Extensive study of linear antenna array synthesis has been reported in the literature [4-6]. For linear array, SLL minimization and null placement can be achieved either by optimizing the excitation amplitude and phase while maintaining uniform spacing as that of conventional array or by optimizing the element spacing while assuming uniform amplitude and phase excitation. Various evolutionary algorithms such as genetic algorithm (GA) [7, 8], simulated annealing (SA) [9], particle swarm optimization (PSO) [10-13], ant colony optimization (ACO) [14], cat swarm optimization (CSO) [15], invasive weed optimization (IWO) [16, 17], Taguchi's method [18], biogeography based optimization [19], artificial bees colony algorithm [20], firefly algorithm [21], cuckoo search algorithm [22], differential search algorithm [23], and backtracking search optimization algorithm [24] have been successfully applied for optimization of linear arrays.

The main aim of this paper is to introduce the grey wolf optimization (GWO) algorithm [25] to the electromagnetics and antenna community. GWO is a metaheuristic algorithm inspired by the leadership hierarchy and hunting mechanism of grey wolves. It was proposed by Mirjalili et al. in 2014 [25]. GWO has been applied to solve practical optimization problems in engineering [25] such as tension/compression spring design, welded beam design, pressure vessel design, and optical buffer design. GWO has also been used in areas like allocation of static synchronous compensator (STATCOM) 


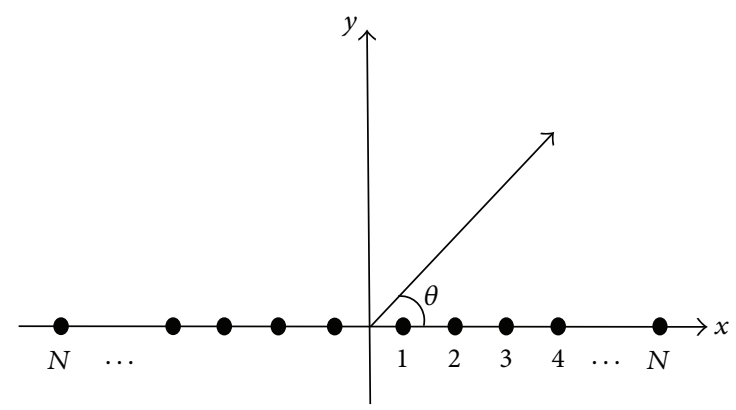

Figure 1: Antenna array geometry.

devices on power system grid [26] and to solve economic dispatch problems [27, 28] and so forth. In [29], an improved grey wolf optimizer for training q-Gaussian Radial Basis Functional-link nets was proposed.

However, this is the first time that GWO is being applied to antenna array optimization to the best of the authors' knowledge. In this paper, GWO has been applied to linear antenna arrays for optimal pattern synthesis in the following two ways: by optimizing the antenna positions while assuming uniform excitation and by optimizing the antenna current amplitudes while assuming spacing and phase as that of uniform array. GWO is used to achieve an array pattern with minimum side lobe level (SLL) along with placement of deep nulls in the specified directions. In addition, GWO is applied for the minimization of the first side lobe nearest to the main beam (near side lobe minimization) while simultaneously controlling the other side lobes.

This section has presented a brief introduction to linear antenna array, the GWO and its applications in optimization problems, and the main objective of this paper. The rest of the paper is organized as follows: the linear antenna array geometry, configuration, and array factor equations are discussed in Section 2. Section 3 presents an elaborate description of the grey wolf optimization algorithm along with a flowchart outlining the steps of GWO implementation. Various examples for linear array synthesis along with the GWO optimized antenna positions and currents as well as the corresponding array patterns are presented in Section 4. The validation of the obtained results by comparison with other nature-inspired evolutionary algorithms is also presented in this section. Section 5 presents the conclusion.

\section{Linear Antenna Array}

A linear antenna array of $2 N$ isotropic elements placed symmetrically along the $x$-axis is considered in this work, as illustrated in Figure 1.

Due to symmetry, the array factor (AF) $[1,10]$ in the azimuth plane is given by

$$
\mathrm{AF}(\theta)=2 \sum_{n=1}^{N} I_{n} \cos \left(k x_{n} \cos (\theta)+\psi_{n}\right),
$$

where $I_{n}, \psi_{n}$, and $x_{n}$ are the excitation amplitude, phase, and position of $n$th element in the array. $k$ is the wave number and is given by $2 \pi / \lambda$ and $\theta$ is the azimuth angle.
For antenna element position optimization, it is assumed that the antenna array is subjected to uniform amplitude and phase excitation; that is, $I_{n}=1$ and $\psi_{n}=0$. Thus, the AF in (1) gets modified to

$$
\operatorname{AF}(\theta)=2 \sum_{n=1}^{N} \cos \left(k x_{n} \cos (\theta)\right) .
$$

The proper placement of antennas in the linear array is very important because if antennas are placed too close to each other, this leads to mutual coupling effects and if antennas are placed too far away, this leads to grating lobes. Thus, conditions (3) must be satisfied for antenna position optimization:

$$
\begin{aligned}
& \left|x_{i}-x_{j}\right|>0.25 \lambda, \\
& \min \left\{x_{i}\right\}>0.125 \lambda ; \quad i=1,2, \ldots, N, i \neq j,
\end{aligned}
$$

where $x_{j}$ is the antenna position adjacent to the antenna position $x_{i}$ and $\left\{x_{i}\right\}$ is the set of all antenna positions.

The array factor for antenna current amplitude optimization is given by (4). Uniform phase, that is, $\psi_{n}=0$, and the interelement spacing $\left(x_{n}\right)$ of $\lambda / 2$ as that of uniform array are assumed:

$$
\operatorname{AF}(\theta)=2 \sum_{n=1}^{N} I_{n} \cos \left(k x_{n} \cos (\theta)\right)
$$

\section{Grey Wolf Optimization (GWO) Algorithm}

GWO is a metaheuristic algorithm which mimics the social hierarchy and hunting mechanism of grey wolves. The hierarchy of grey wolves is as follows: the leaders are male and female, called alphas $(\alpha)$. The second level in the hierarchy comprises the beta $(\beta)$ wolves followed by delta $(\delta)$ wolves and the lowest ranking grey wolves are the omegas $(\omega)$. The hierarchy of grey wolves is mathematically modelled by considering $\alpha$ as the fittest solution followed by the second and third best solutions, $\beta$ and $\delta$, respectively. The rest of the candidate solutions are assumed to be $\omega$.

The main phases of grey wolf hunting are as follows [30]:

(1) Tracking, chasing, and approaching the prey.

(2) Pursuing, encircling, and harassing the prey until it stops moving.

(3) Attack towards the prey.

The encircling behavior is mathematically modelled by (5) as in [25]:

$$
\begin{aligned}
\vec{D} & =\left|\vec{C} \cdot \overrightarrow{X_{p}(t)}-\overrightarrow{X(t)}\right|, \\
\vec{X}(t+1) & =\overrightarrow{X_{p}}(t)-\vec{A} \cdot(\vec{D}),
\end{aligned}
$$

where $t$ indicates the current iteration, $\vec{A}$ and $\vec{C}$ are coefficient vectors, $\overrightarrow{X_{p}}$ is the position vector of the prey, and $\vec{X}$ indicates 
the position vector of a grey wolf. The vectors $\vec{A}$ and $\vec{C}$ are calculated using

$$
\begin{aligned}
& \vec{A}=2 \vec{a} \cdot \overrightarrow{r_{1}}-\vec{a}, \\
& \vec{C}=2 \cdot \overrightarrow{r_{2}},
\end{aligned}
$$

where components of $\vec{a}$ are linearly decreased from 2 to 0 over the course of iterations and $\overrightarrow{r_{1}}, \overrightarrow{r_{2}}$ are random vectors in $[0,1]$.

In the GWO algorithm, the hunting (optimization) is guided by $\alpha, \beta$, and $\delta$. The $\omega$ wolves follow these three wolves. The $\alpha, \beta$, and $\delta$ wolves are supposed to have better knowledge about the potential location of prey. Therefore, the first three best solutions are saved and the other search agents update their positions according to the position of the best search agent. The following equations are used for this purpose [25]:

$$
\begin{aligned}
\overrightarrow{D_{\alpha}} & =\left|\overrightarrow{C_{1}} \cdot \overrightarrow{X_{\alpha}}-\vec{X}\right|, \\
\overrightarrow{D_{\beta}} & =\left|\overrightarrow{C_{2}} \cdot \overrightarrow{X_{\beta}}-\vec{X}\right|, \\
\overrightarrow{D_{\delta}} & =\left|\overrightarrow{C_{3}} \cdot \overrightarrow{X_{\delta}}-\vec{X}\right|, \\
\overrightarrow{X_{1}} & =\overrightarrow{X_{\alpha}}-\overrightarrow{A_{1}} \cdot\left(\overrightarrow{D_{\alpha}}\right), \\
\overrightarrow{X_{2}} & =\overrightarrow{X_{\beta}}-\overrightarrow{A_{2}} \cdot\left(\overrightarrow{D_{\beta}}\right), \\
\overrightarrow{X_{3}} & =\overrightarrow{X_{\delta}}-\overrightarrow{A_{3}} \cdot\left(\overrightarrow{D_{\delta}}\right), \\
\vec{X}(t+1) & =\frac{\overrightarrow{X_{1}}+\overrightarrow{X_{2}}+\overrightarrow{X_{3}}}{3} .
\end{aligned}
$$

By using (7), a search agent updates its position according to $\alpha, \beta$, and $\delta$ in the $n$-dimensional search space as depicted in Figure 2(a) [25]. Furthermore, the final position would be in a random place in the search space within a circle which is defined by the positions of $\alpha, \beta$, and $\delta$. Hence, $\alpha, \beta$, and $\delta$ estimate the position of the prey, and other wolves update their positions randomly around the prey.

The following steps summarize the GWO algorithm:

(1) Create a random population of grey wolves (candidate solutions).

(2) $\alpha, \beta$, and $\delta$ wolves estimate the probable position of the prey over the course of iterations. Each candidate solution updates its distance from the prey.

(3) The parameter " $\vec{a}$ " is decreased linearly from 2 to 0 in order to emphasize exploration (searching for prey) and exploitation (attacking the prey), respectively. Candidate solutions tend to diverge from the prey when $|\vec{A}|>1$ and converge towards the prey when $|\vec{A}|<1$ so as to avoid stagnation in local solutions.

(4) Finally, the GWO algorithm is terminated by the satisfaction of an end criterion.

The flowchart of GWO is depicted in Figure 2(b).
TABLE 1: Optimized positions of the positive half of the 10-element array of Example 1.

\begin{tabular}{llllll}
\hline Method & \multicolumn{5}{c}{ Optimized element positions } \\
\hline CSO [15] & $0.1516 \lambda$ & $0.4115 \lambda$ & $0.7899 \lambda$ & $1.1048 \lambda$ & $1.6843 \lambda$ \\
Proposed & $0.1250 \lambda$ & $0.3680 \lambda$ & $0.7522 \lambda$ & $0.9851 \lambda$ & $1.5546 \lambda$ \\
\hline
\end{tabular}

\section{Results and Discussion}

In this section, the GWO algorithm is applied to linear antenna array for optimal pattern synthesis. The GWO is implemented on MATLAB $^{\circledR}$ and executed 15 times. The number of iterations for each run is set equal to 1000. All results were obtained using grey wolf population $(n)$ equal to 30 .

4.1. Antenna Position Optimization. This section presents Examples 1-3 for optimization of the antenna positions $\left(x_{n}\right)$ in order to achieve a desired array pattern with minimum SLL and null positioning. Uniform amplitude and phase excitations are assumed; that is, $I_{n}=1$ and $\psi_{n}=0$.

4.1.1. Peak SLL Minimization. The fitness function used for the minimization of peak SLL is formulated using $(8)[13,19$, 22]. Consider

$$
\text { Fitness }=\min (\max (20 \log |\mathrm{AF}(\theta)|)),
$$

where $\theta \in$ spatial region in which SLL is to suppressed and $\operatorname{AF}(\theta)$ is the array factor given by (2).

Example 1 illustrates the design of $2 N=10$ element linear array for achieving minimum SLL in the regions, $\theta=$ $\left[0^{\circ}, 74^{\circ}\right]$ and $\theta=\left[106^{\circ}, 180^{\circ}\right]$. The grey wolf optimization algorithm with fitness function given by (8) is used for the determination of optimized element locations, $x_{n}$. Uniform amplitude and phase excitations are assumed; that is, $I_{n}=1$ and $\psi_{n}=0$. The optimized element positions are shown in Table 1 and the array pattern is illustrated in Figure 3. For benchmarking, the peak SLL obtained for this example using the proposed method (GWO) and other nature-inspired optimization techniques is summarized in Table 2. There is an improvement in the reduction of SLL using the proposed approach (GWO) as compared to conventional array as well as arrays optimized using other optimization algorithms such as ACO [14], PSO [15], and CSO [15]. The proposed method (GWO) gives peak SLL of $-23.42 \mathrm{~dB}$ which is $10.19 \mathrm{~dB}$ lower as compared to conventional array. The peak SLL has been lowered from $-20.72 \mathrm{~dB}$ to $-23.42 \mathrm{~dB}$ (by $2.7 \mathrm{~dB}$ ) as compared to PSO optimized array, from $-22.66 \mathrm{~dB}$ to $-23.42 \mathrm{~dB}$ as compared to ACO optimized array, and from $-22.89 \mathrm{~dB}$ to $-23.42 \mathrm{~dB}$ as compared to CSO optimized array.

4.1.2. SLL Minimization along with Null Placement. The fitness function as stated in (9) is used for SLL minimization as well as for placement of nulls in desired directions [10, 19]:

$$
\text { Fitness }=\sum_{i} \frac{1}{\Delta \theta_{i}} \int_{\theta_{l i}}^{\theta_{u i}}|\operatorname{AF}(\theta)|^{2} d \theta+\sum_{k}\left|\operatorname{AF}\left(\theta_{k}\right)\right|^{2},
$$




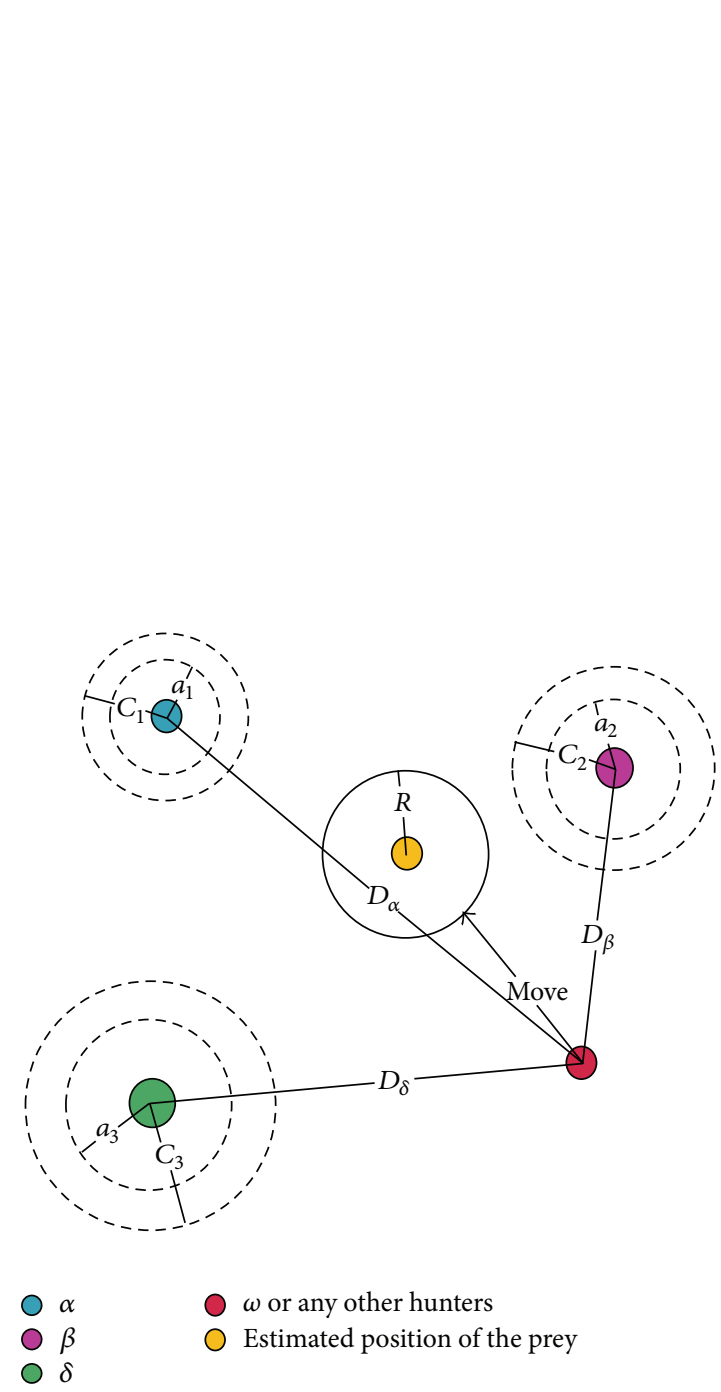

(a)

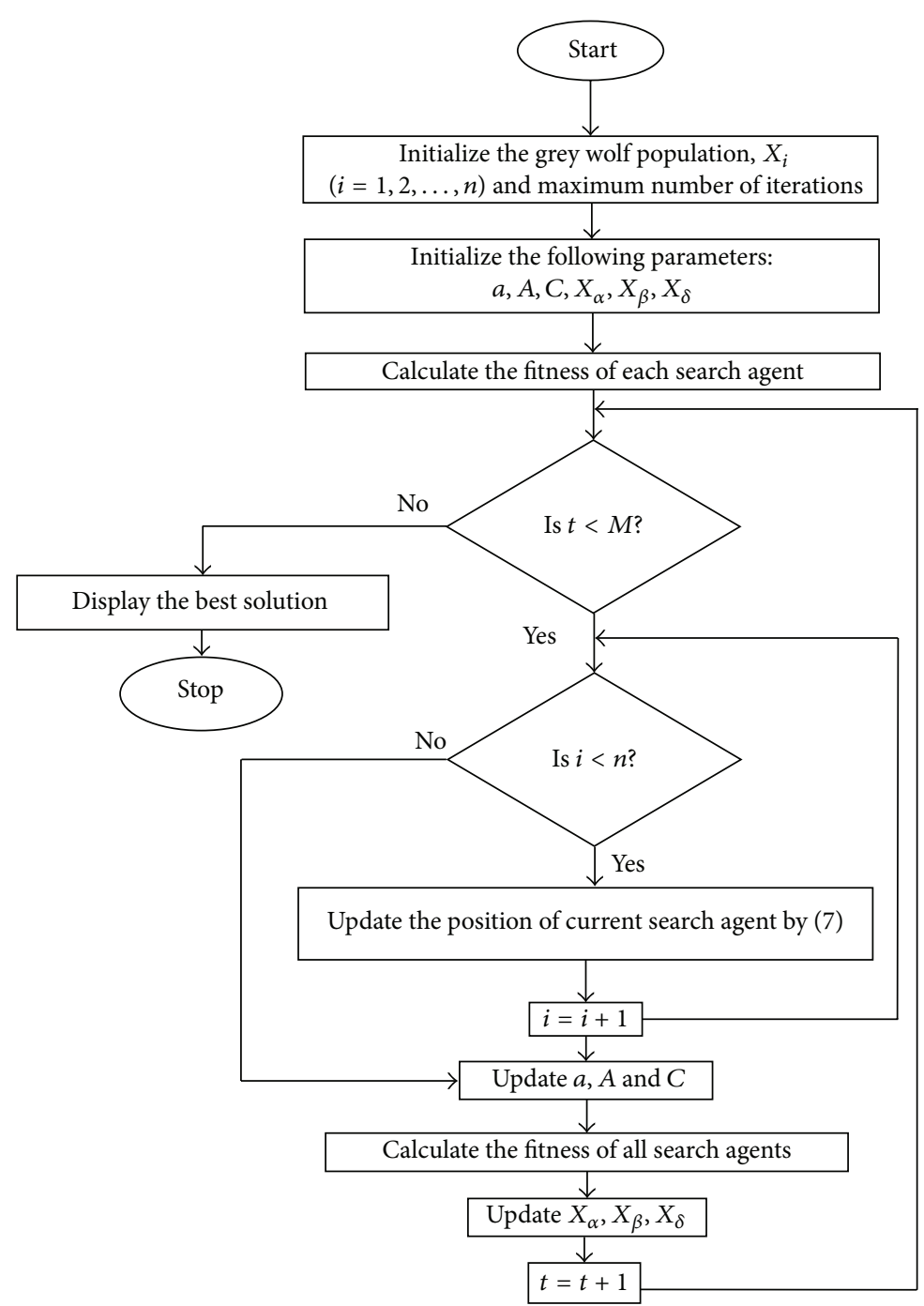

(b)

Figure 2: (a) Position update in GWO [25]. (b) Flowchart of the grey wolf optimization algorithm.

where $\theta_{l i}$ and $\theta_{u i}$ are the spatial regions in which SLL is suppressed and $\Delta \theta_{i}=\theta_{u i}-\theta_{l i}$. The null direction is given by $\theta_{k}$. In (9), the first term of the fitness function is for SLL suppression and the second term accounts for the placement of nulls in the desired directions. $\operatorname{AF}(\theta)$ is the array factor given by (2).

Example 2 illustrates the synthesis of 28-element linear antenna array for achieving SLL minimization in the regions $\theta=\left[0^{\circ}, 84^{\circ}\right]$ and $\theta=\left[96^{\circ}, 180^{\circ}\right]$ along with null placement at $\theta=55^{\circ}, 57.5^{\circ}, 60^{\circ}, 120^{\circ}, 122.5^{\circ}$, and $125^{\circ}$. The fitness function used by the GWO for this example is given by (9). The array pattern is shown in Figure 4 and the optimized positions of the antenna elements are given in Table 3. From Table 3 , it is seen that the array optimized using the proposed method (GWO) and CSO [15] optimized array have almost the same length. It is deduced from Figure 4 that the proposed method using GWO enables the placement of nulls, as deep as $-84.42 \mathrm{~dB}$ at the desired directions.
TABLE 2: Optimized peak SLL for 10-element linear array of Example 1 .

\begin{tabular}{lcc}
\hline Serial number & Approach & Peak SLL (in dB) \\
\hline 1 & $\begin{array}{c}\text { Conventional (without } \\
\text { optimization) [15] }\end{array}$ & -13.23 \\
2 & PSO [15] & -20.72 \\
3 & ACO [14] & -22.66 \\
4 & CSO [15] & -22.89 \\
5 & Proposed & -23.42 \\
\hline
\end{tabular}

The null depths obtained by the proposed method using GWO at each of the specified directions are summarized in Table 4 . The comparative analysis of null depth obtained with the proposed method using GWO and various other stateof-the-art optimization algorithms is shown in Table 5. It is 
TABLE 3: Optimized positions of the positive half of the 28-element array of Example 2.

\begin{tabular}{llllllll}
\hline Method & \multicolumn{7}{c}{ Optimized element positions } \\
\hline \multirow{2}{*}{ CSO [15] } & $0.2720 \lambda$ & $0.7547 \lambda$ & $1.1399 \lambda$ & $1.7065 \lambda$ & $2.3287 \lambda$ & $2.8675 \lambda$ & $3.3536 \lambda$ \\
& $3.7693 \lambda$ & $4.2222 \lambda$ & $4.8991 \lambda$ & $5.4061 \lambda$ & $5.7389 \lambda$ & $6.1564 \lambda$ & $6.7173 \lambda$ \\
\hline \multirow{2}{*}{ Proposed } & $0.2298 \lambda$ & $0.7266 \lambda$ & $1.1376 \lambda$ & $1.7300 \lambda$ & $2.1831 \lambda$ & $2.6710 \lambda$ \\
& $3.4867 \lambda$ & $3.7652 \lambda$ & $4.3933 \lambda$ & $4.9039 \lambda$ & $5.3422 \lambda$ & $5.7610 \lambda$ & $6.3378 \lambda$ \\
\hline
\end{tabular}

TABLE 4: Null depths after optimization by GWO algorithm for Example 2.

\begin{tabular}{lcccccc}
\hline $\begin{array}{l}\text { Linear array } \\
\text { type }\end{array}$ & $55^{\circ}$ & $57.5^{\circ}$ & $60^{\circ}$ & $120^{\circ}$ & $122.5^{\circ}$ & $125^{\circ}$ \\
\hline $\begin{array}{l}\text { 28-element } \\
\text { array }\end{array}$ & -83.22 & -84.42 & -78.41 & -78.41 & -84.42 & -83.22 \\
\hline
\end{tabular}

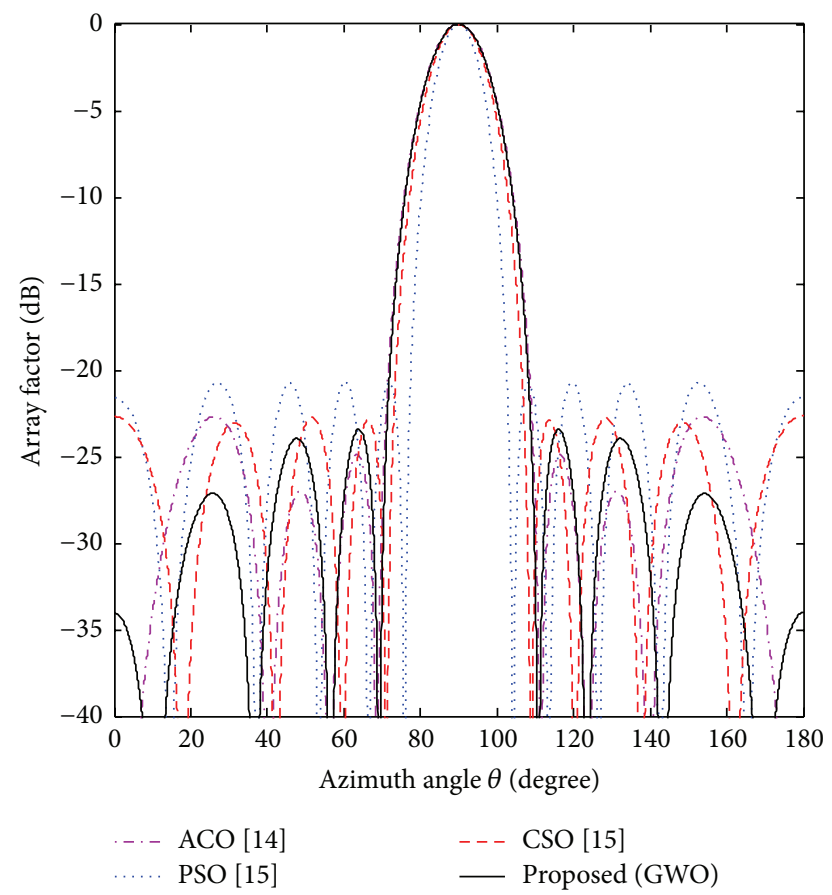

Figure 3: Array pattern for Example 1.

seen that, for this example, the proposed method using GWO places the deepest null of $-84.42 \mathrm{~dB}$. There is an improvement of around $34 \mathrm{~dB}$ over the null depth obtained by using PSO [15] and ACO [14]. As compared to CSO [15], the proposed approach of using GWO gives improvement of around $19 \mathrm{~dB}$ in null depth. However, the SLL obtained is similar for all the above algorithms.

Example 3 illustrates the application of GWO to optimize the antenna element positions for SLL minimization and null placement of 32-element linear antenna array. The fitness function used by the GWO for this example is given by (9). SLL reduction is desired in the spatial regions $\theta=\left[0^{\circ}, 85^{\circ}\right]$ and $\theta=\left[95^{\circ}, 180^{\circ}\right]$ whereas nulls are desired at $\theta=81^{\circ}$ and $\theta=99^{\circ}$ (very close to the first side lobe). The array pattern is shown in Figure 5 and the optimized positions of the antenna
TABLE 5: Comparative analysis of null depth (in $\mathrm{dB}$ ) obtained by various optimization algorithms for Example 2.

\begin{tabular}{lcccc}
\hline & \multicolumn{4}{c}{ Method } \\
& PSO [15] & ACO [14] & CSO [15] & Proposed \\
\hline $\begin{array}{l}\text { Null depth } \\
\text { (in dB) }\end{array}$ & -50 & $\sim-50$ & -65 & $\mathbf{- 8 4 . 4 2}$ \\
\hline
\end{tabular}

TABLE 6: Optimized positions of the positive half of the 32-element array of Example 3.

\begin{tabular}{lcccc}
\hline Method & \multicolumn{4}{c}{ Optimized element positions } \\
\hline \multirow{4}{*}{ CSO [15] } & $0.2883 \lambda$ & $0.6830 \lambda$ & $1.1929 \lambda$ & $1.5199 \lambda$ \\
& $3.9768 \lambda$ & $2.3247 \lambda$ & $2.6886 \lambda$ & $3.1362 \lambda$ \\
& $5.4817 \lambda$ & $3.9538 \lambda$ & $4.3822 \lambda$ & $4.9252 \lambda$ \\
& $0.194307 \lambda$ & $6.2091 \lambda$ & $7.0412 \lambda$ & $7.7500 \lambda$ \\
\hline Proposed & $2.241275 \lambda$ & $2.714261 \lambda$ & $2.999822 \lambda$ & $3.451514 \lambda$ \\
& $3.753935 \lambda$ & $4.275930 \lambda$ & $4.75 \lambda$ & $5.255635 \lambda$ \\
& $5.751775 \lambda$ & $6.455911 \lambda$ & $7.25 \lambda$ & $8.0 \lambda$ \\
\hline
\end{tabular}

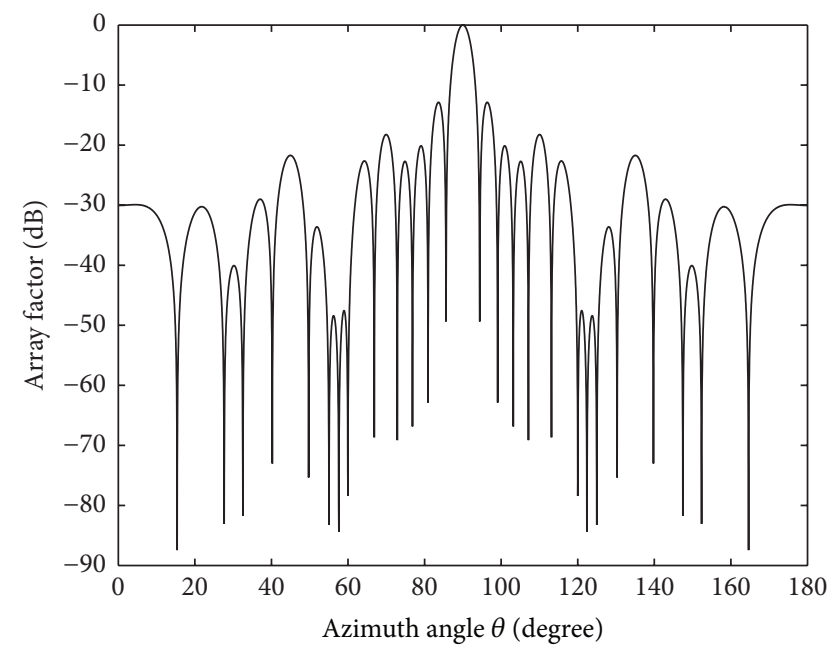

FIGURE 4: Array pattern for Example 2.

elements are given in Table 6. The array optimized by the proposed approach of using GWO has almost the same length as that obtained by CSO [15]. It is seen from Figure 5 that the proposed approach of using GWO enables the placement of nulls as deep as $-106 \mathrm{~dB}$ at the desired directions $\left(\theta=81^{\circ}\right.$ and $\theta=99^{\circ}$ ), very close to the first side lobe.

For this example, PSO [15] offers null depth of $-60 \mathrm{~dB}$ and ACO [14] gives $-50 \mathrm{~dB}$ nulls whereas CSO [15] places deep 
TABLE 7: Comparative analysis of null depth (in $\mathrm{dB}$ ) obtained by various optimization algorithms for Example 3.

\begin{tabular}{lcccc}
\hline & \multicolumn{4}{c}{ Method } \\
& PSO [15] & ACO [14] & CSO [15] & Proposed \\
\hline Null depth (in dB) & -60 & -50 & -80 & $-\mathbf{1 0 6}$ \\
\hline
\end{tabular}

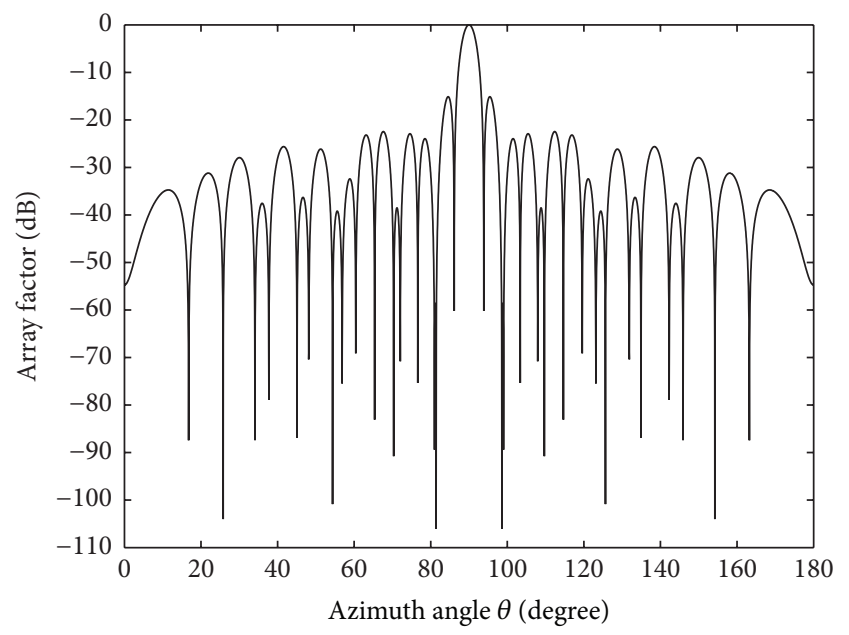

FIgURE 5: Array pattern for Example 3.

nulls of $-80 \mathrm{~dB}$ as seen from Table 7 . However, the proposed approach (GWO) places the deepest null of $-106 \mathrm{~dB}$. The first side lobe obtained by GWO is about $3 \mathrm{~dB}$ higher than that obtained using CSO [15], but the remaining side lobes are almost similar (some are even lower) to those obtained by using CSO [15].

4.1.3. Near SLL Minimization. Minimization of the first side lobe nearest to the main lobe (near side lobe) by the optimization of antenna positions is illustrated in Example 4. The fitness function mentioned in (10) is used for this purpose [13]. Consider

$$
\begin{aligned}
& \text { Fitness }=\min \left(\alpha_{1} \max \left\{20 \log \left|\operatorname{AF}\left(\theta_{\mathrm{AS}}\right)\right|\right\}\right. \\
& \left.+\alpha_{2} \max \left\{20 \log \left|\operatorname{AF}\left(\theta_{\mathrm{NS}}\right)\right|\right\}\right),
\end{aligned}
$$

where $\theta_{\mathrm{AS}}=\left[0^{\circ}, 76^{\circ}\right]$ and $\left[104^{\circ}, 180^{\circ}\right]$ and $\theta_{\mathrm{NS}}=\left[69^{\circ}, 76^{\circ}\right]$ and $\left[104^{\circ}, 111^{\circ}\right] . \theta_{\mathrm{NS}}$ specifies the near side lobe region and its weight is $\alpha_{2}$. The near side lobe region can be controlled by using the fitness function given by (10) and the amount of its reduction is controlled by the weights $\alpha_{1}$ and $\alpha_{2}$. For illustration of near SLL suppression, design of Example 4 is presented in which a 10-element linear array is optimized using (10) and weights $\alpha_{1}=1$ and $\alpha_{2}=2$. The optimized antenna positions are given in Table 8 . It is seen that the length of array optimized by the proposed approach is the same as that of PSO [13] optimized array. The array pattern is shown in Figure 6.

The near SLL obtained by GWO is $-31.3 \mathrm{~dB}$ which is $18.33 \mathrm{~dB}$ lower as compared to uniform array. The peak SLL is found to be $-18.34 \mathrm{~dB}$. The benchmarking results are shown in Table 9.
TABLE 8: Optimized positions of the positive half of the 10-element array of Example 4.

\begin{tabular}{llllll}
\hline Method & \multicolumn{5}{c}{ Optimized element positions } \\
\hline PSO [13] & $0.1685 \lambda$ & $0.5461 \lambda$ & $0.9364 \lambda$ & $1.5107 \lambda$ & $2.2500 \lambda$ \\
Proposed & $0.1792 \lambda$ & $0.5410 \lambda$ & $0.9354 \lambda$ & $1.5096 \lambda$ & $2.2500 \lambda$ \\
\hline
\end{tabular}

TABLE 9: Near SLL and peak SLL for Example 4.

\begin{tabular}{lcc}
\hline & Near SLL $(\mathrm{dB})$ & Peak SLL $(\mathrm{dB})$ \\
\hline Uniform array & -12.97 & -12.97 \\
PSO [13] & -31 & -18.3 \\
Proposed & -31.3 & -18.34 \\
\hline
\end{tabular}

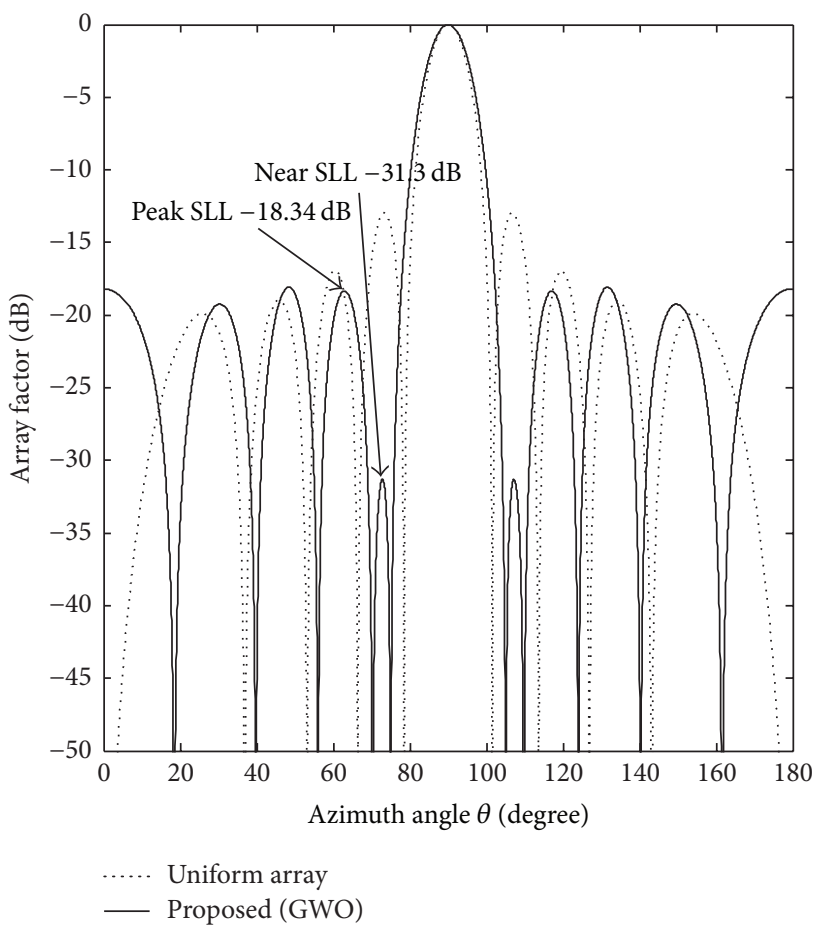

FiguRE 6: Array pattern for Example 4.

4.2. Antenna Current Optimization. In this section, Examples 5-8 are presented for optimization of the antenna current amplitudes $\left(I_{n}\right)$ for optimal pattern synthesis. Handling phase involves a huge complexity as it is not always convenient to implement fractional phase in practice. Hence, uniform phase excitation is assumed; that is, $\psi_{n}=0$. The element spacing of $\lambda / 2$ is considered which is the same as that for uniform array.

4.2.1. Peak SLL Minimization. This section presents Examples 5 and 6 which illustrate the application of GWO for optimization of antenna current amplitudes in order to minimize the peak SLL in the regions, $\theta=\left[0^{\circ}, 76^{\circ}\right]$ and $\theta=$ $\left[104^{\circ}, 180^{\circ}\right]$. The fitness function given by (8) is used for both the examples. The optimized current amplitudes obtained by using the proposed method are shown in Table 10. The array patterns for Examples 5 and 6 are illustrated in Figures 7 and 8 , respectively. The current amplitude distribution along the 
TABLE 10: Optimized current amplitudes for peak SLL minimization.

\begin{tabular}{lcc}
\hline Linear array type & Method & Optimized current amplitudes \\
\hline \multirow{2}{*}{10 elements (Example 5) } & PSO [13] & $1.0000,0.9010,0.7255,0.5120,0.4088$ \\
& Proposed & $1.0000,0.8962,0.6963,0.4935,0.2964$ \\
\hline \multirow{2}{*}{16 elements (Example 6) } & PSO [13] & $1.0000,0.9521,0.8605,0.7372,0.5940,0.4465,0.3079,0.2724$ \\
& Proposed & $1.0000,0.9339,0.8127,0.6533,0.4855,0.3195,0.1928,0.1002$ \\
\hline
\end{tabular}

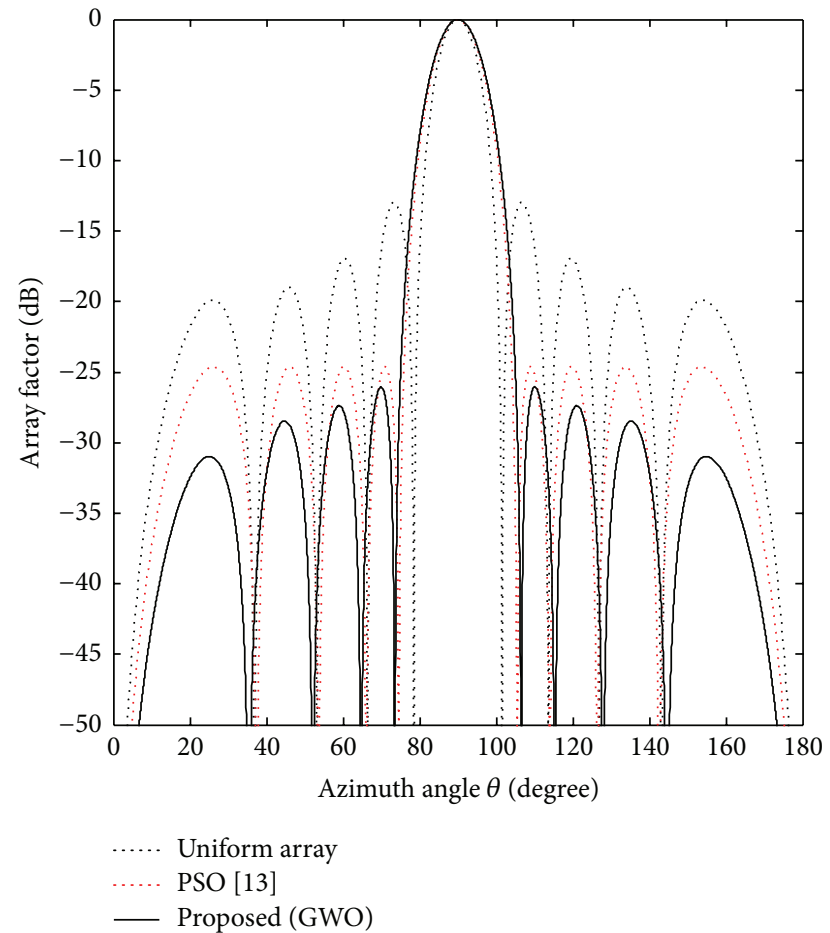

Figure 7: Array pattern for Example 5.

array elements is shown in Figures 9(a) and 9(b) for Examples 5 and 6 , respectively. The current amplitude is maximum at the centre of the array and decreases from the centre towards the edge elements. Such an amplitude distribution makes it feasible to use power dividers.

For Example 5, the proposed method (GWO) gives peak SLL of $-26.05 \mathrm{~dB}$ which is $13.08 \mathrm{~dB}$ lower as compared to uniform array. The peak SLL has been lowered from $-24.62 \mathrm{~dB}$ to $-26.05 \mathrm{~dB}$ (by $1.43 \mathrm{~dB}$ ) as compared to PSO [13] optimized array as depicted in Figure 7 and summarized in Table 11.

The peak SLL obtained by the proposed method for Example 6 is $-40.5 \mathrm{~dB}$ which is $27.35 \mathrm{~dB}$ lower as compared to uniform array. The peak SLL has been lowered from $-30.7 \mathrm{~dB}$ to $-40.5 \mathrm{~dB}$ as compared to PSO [13] optimized array. These results are depicted in Figure 8 and summarized in Table 11.

4.2.2. SLL Minimization along with Null Placement. To illustrate this case, Example 7 presents the synthesis of 20-element linear array for achieving SLL minimization in the regions $\theta=\left[0^{\circ}, 82^{\circ}\right]$ and $\theta=\left[98^{\circ}, 180^{\circ}\right]$ along with null placement at $\theta=64^{\circ}, 76^{\circ}, 104^{\circ}$, and $116^{\circ}$. The fitness function used for this
TABLE 11: Benchmarking results for peak SLL minimization.

\begin{tabular}{lcc}
\hline & \multicolumn{2}{c}{ Peak SLL (dB) } \\
& Example 5 & Example 6 \\
\hline Uniform array (without optimization) & -12.97 & -13.15 \\
PSO [13] & -24.62 & -30.7 \\
Proposed & -26.05 & -40.5 \\
\hline
\end{tabular}

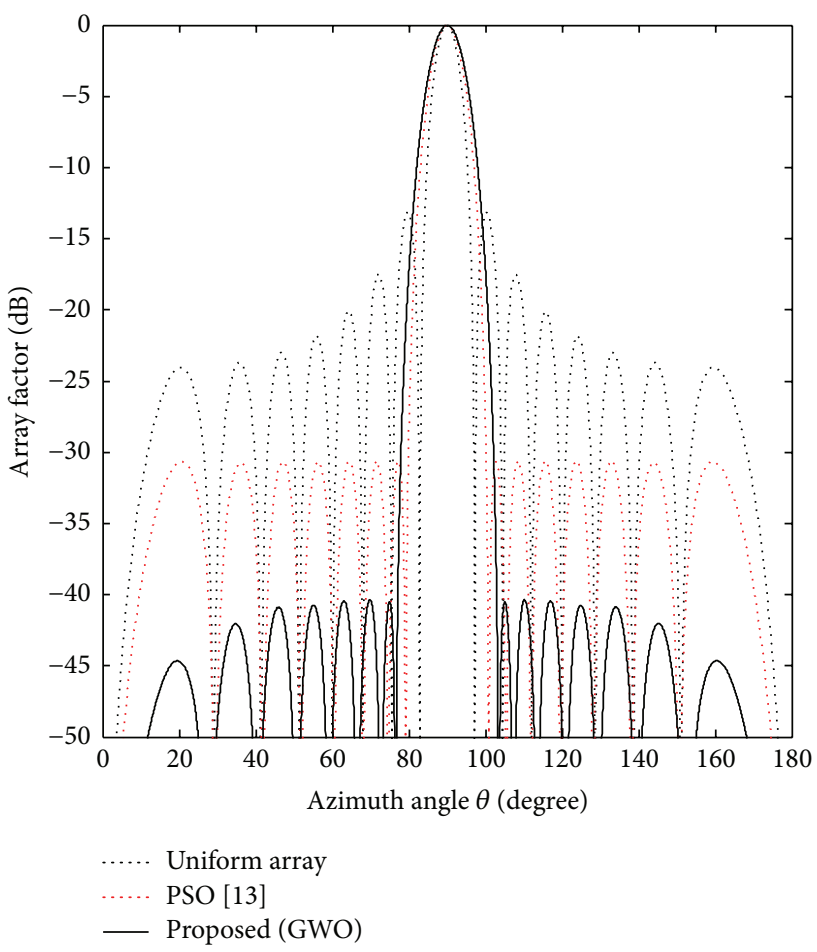

Figure 8: Array pattern for Example 6.

example is given by (9). The optimized current amplitudes are given in Table 12 and the array pattern is shown in Figure 10(a). The current amplitude is maximum at the centre of the array and decreases from the centre towards the edge elements as shown in Figure 10(b).

The proposed method enables the placement of nulls (as deep as $-92.02 \mathrm{~dB}$ ) in the desired directions. The comparative analysis of peak SLL and null depths obtained by using the proposed method (GWO) and those obtained by uniform array and by using BBO [19] is shown in Table 13.

It is seen that the peak SLL obtained by using the proposed approach is $-28.44 \mathrm{~dB}$ which is $15.25 \mathrm{~dB}$ lower than uniform array and $1.73 \mathrm{~dB}$ lower than BBO [19] array. Also, the null depths obtained by using the proposed method are 
TABLE 12: Optimized current amplitudes of the positive half of the 20-element array of Example 7.

\begin{tabular}{|c|c|c|c|c|c|c|c|c|c|c|}
\hline \multirow{2}{*}{$\begin{array}{l}\text { Method } \\
\text { BBO [19] }\end{array}$} & \multicolumn{10}{|c|}{ Optimized current amplitudes } \\
\hline & 1.0000 & 0.9747 & 0.9264 & 0.8575 & 0.7022 & 0.6242 & 0.4799 & 0.3607 & 0.2369 & 0.1234 \\
\hline Proposed & 1.0000 & 0.9794 & 0.9254 & 0.8126 & 0.7008 & 0.6000 & 0.4594 & 0.3326 & 0.2133 & 0.1167 \\
\hline
\end{tabular}

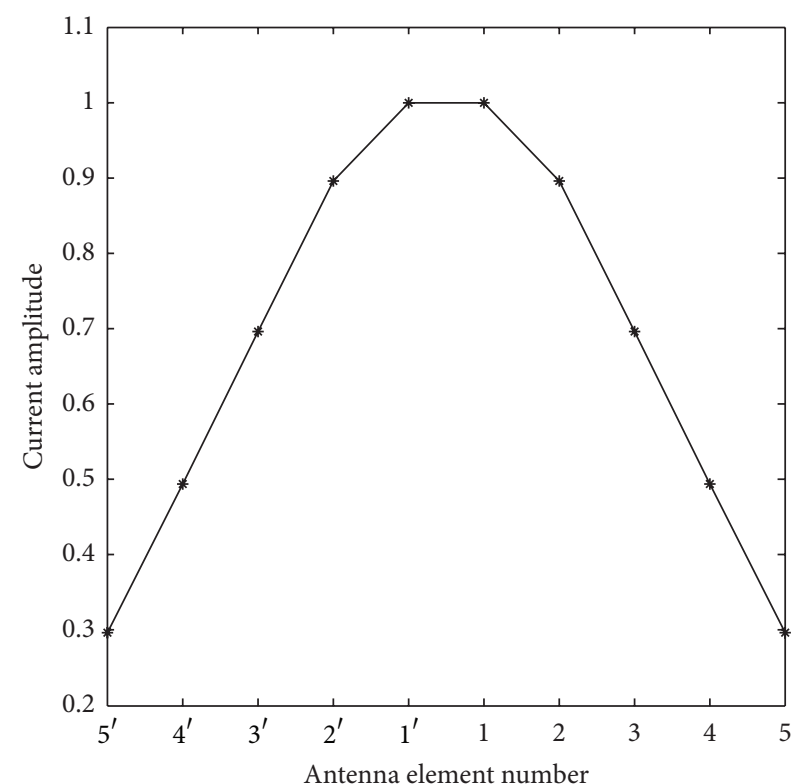

(a)

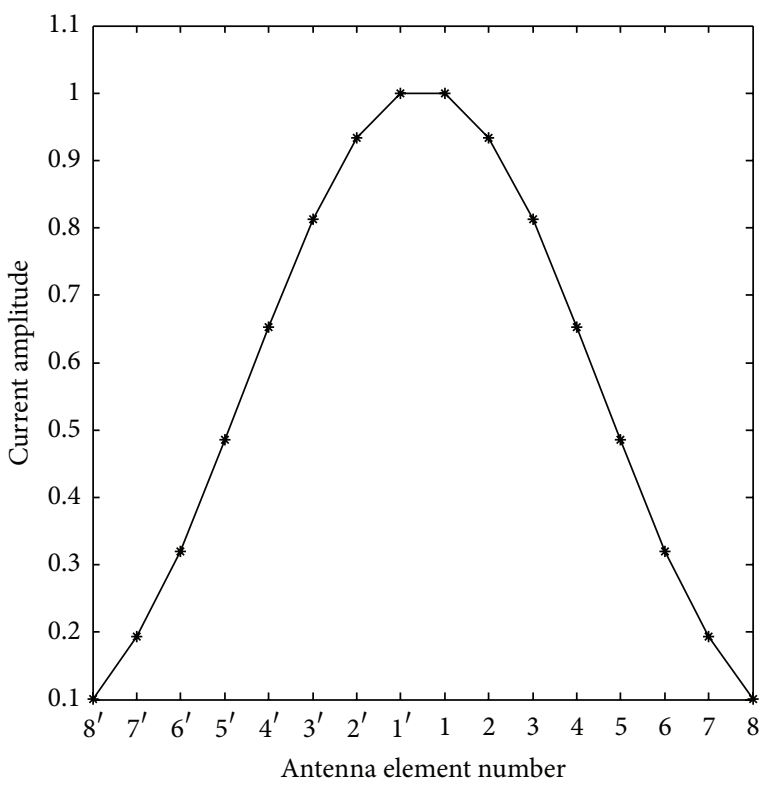

(b)

FIGURE 9: Current distribution for (a) Example 5 and (b) Example 6.

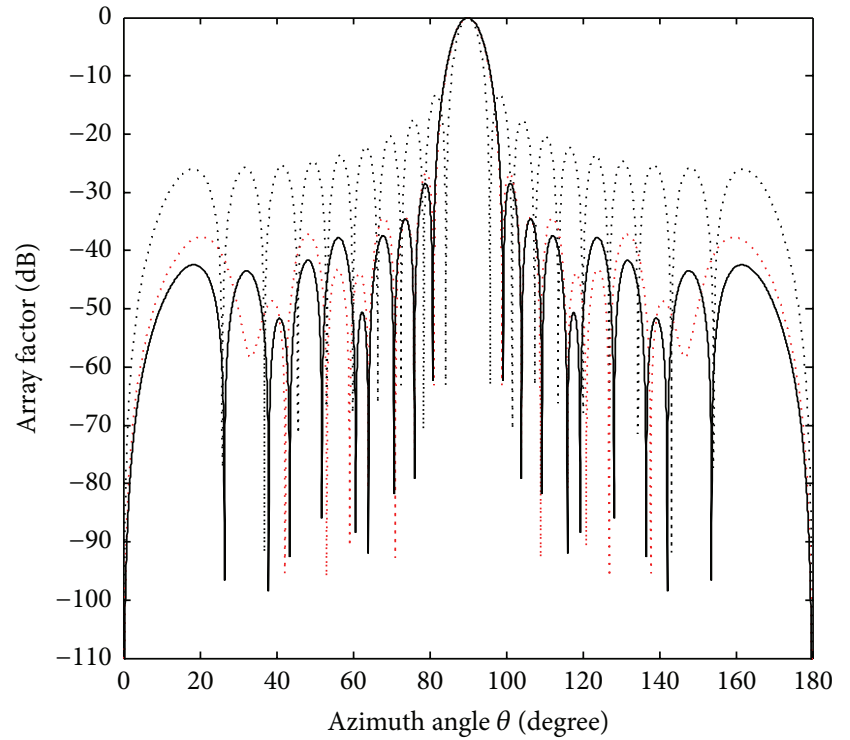

Uniform array

BBO [19]

— Proposed (GWO)

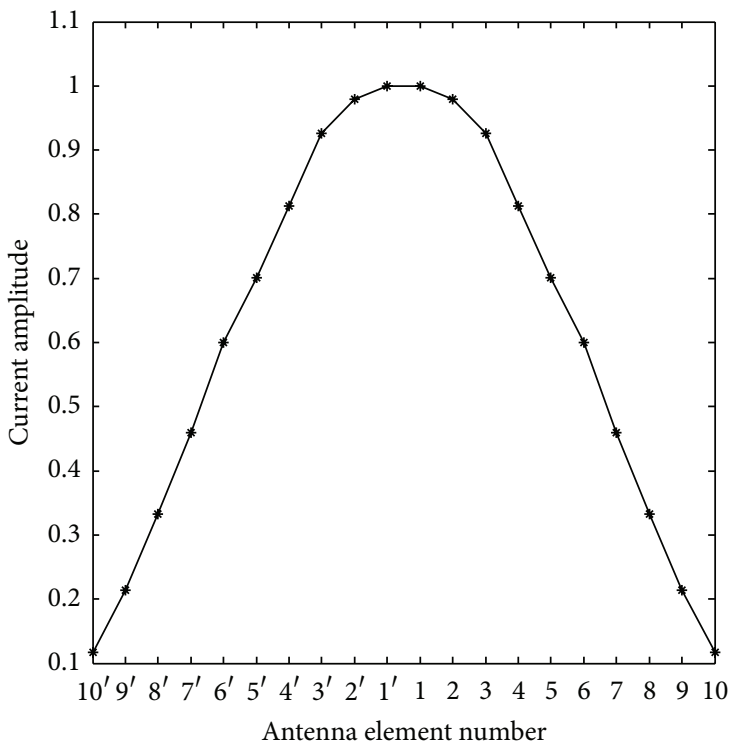

(a)

(b)

FIgURE 10: Example 7: (a) array pattern and (b) current distribution. 
TABLE 13: Peak SLL and null depths for Example 7.

\begin{tabular}{lccccc}
\hline & \multirow{2}{*}{ Peak SLL (dB) } & \multicolumn{4}{c}{ Null depth (dB) } \\
& & $64^{\circ}$ & $76^{\circ}$ & $104^{\circ}$ & $116^{\circ}$ \\
\hline Uniform array & -13.19 & -22.7 & -17.7 & -17.7 & -22.7 \\
BBO [19] & -26.71 & -79.66 & -74.2 & -74.2 & -79.66 \\
Proposed & -28.44 & -92.02 & -79.12 & -79.12 & -92.02 \\
\hline
\end{tabular}

TABLE 14: Optimized current amplitudes of the positive half of the 10-element array of Example 8.

\begin{tabular}{lcllll}
\hline Method & \multicolumn{5}{l}{ Optimized current amplitudes } \\
\hline BBO [19] & 1.0000 & 0.8526 & 0.6586 & 0.4601 & 0.5101 \\
Proposed & 1.000 & 0.8169 & 0.6383 & 0.4505 & 0.4505 \\
\hline
\end{tabular}

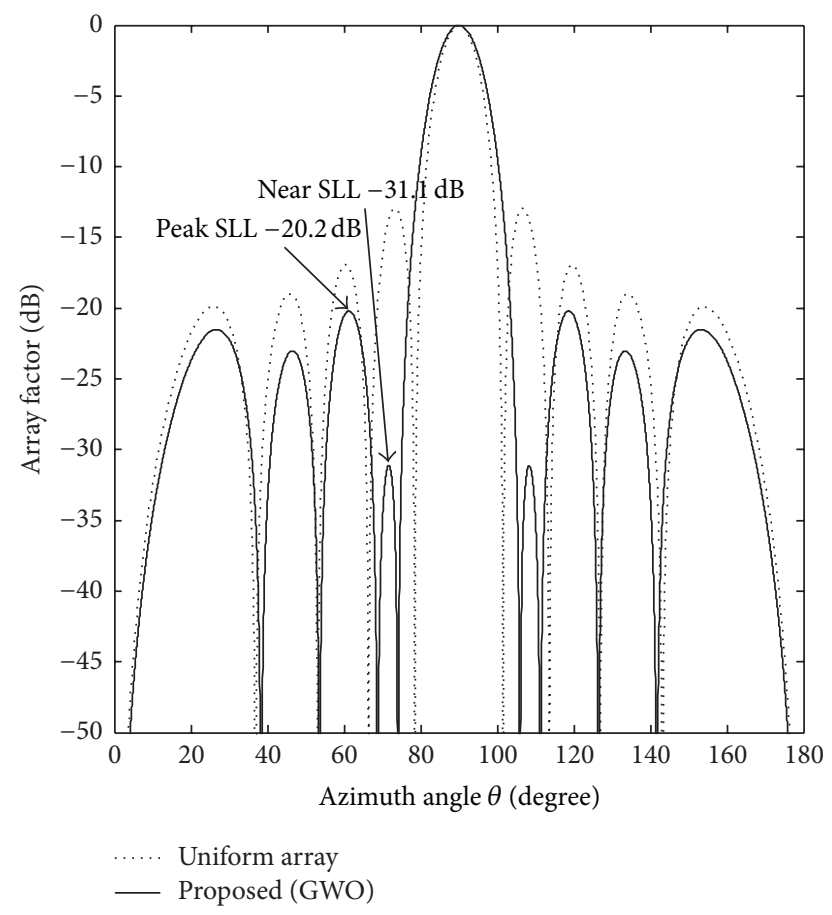

FIgURE 11: Array pattern for Example 8.

much higher than that obtained by using BBO array. The benchmarking results are summarized in Table 13.

4.2.3. Near SLL Minimization. Minimization of the near side lobe of a 10-element array by the optimization of antenna current amplitudes is illustrated in Example 8. The fitness function given by $(10)$ is used for this purpose with $\theta_{\mathrm{AS}}=$ $\left[0^{\circ}, 76^{\circ}\right]$ and $\left[104^{\circ}, 180^{\circ}\right], \theta_{\mathrm{NS}}=\left[69^{\circ}, 76^{\circ}\right]$ and $\left[104^{\circ}, 111^{\circ}\right]$, and weights $\alpha_{1}=1$ and $\alpha_{2}=2$. The optimized antenna current amplitudes are given in Table 14 and the array pattern is shown in Figure 11.

The near SLL obtained by GWO is $-31.1 \mathrm{~dB}$ which is $18.13 \mathrm{~dB}$ lower as compared to uniform array and $2.51 \mathrm{~dB}$ lower than BBO [19] optimized array. The peak SLL is $-20.2 \mathrm{~dB}$ which shows that GWO enables reduction of the
TABLE 15: Near SLL and peak SLL for Example 8.

\begin{tabular}{lcc}
\hline Method & Near SLL $(\mathrm{dB})$ & Peak SLL $(\mathrm{dB})$ \\
\hline Uniform array & -12.97 & -12.97 \\
BBO [19] & -28.59 & -20.1 \\
Proposed & -31.1 & -20.2 \\
\hline
\end{tabular}

TABLE 16: Computational time for GWO as compared to other optimization algorithms.

\begin{tabular}{|c|c|c|c|}
\hline \multirow{2}{*}{$\begin{array}{l}\text { Linear antenna } \\
\text { array }\end{array}$} & \multicolumn{3}{|c|}{ Computational time for each run (in seconds) } \\
\hline & PSO [15] & CSO [15] & GWO \\
\hline 10 elements & 17 & 101 & 26 \\
\hline 28 elements & 35 & 246 & 44 \\
\hline
\end{tabular}

near side lobe while simultaneously controlling the other side lobes. The benchmarking results are shown in Table 15 .

4.3. Computational Time. The computational time required for GWO to converge to the optimum solution as compared to other state-of-the-art algorithms is shown in Table 16. All the computations are performed using MATLAB on a PC operating at $3 \mathrm{GHz}$ with $2 \mathrm{~GB}$ of RAM.

It is seen that GWO converges to the optimum solution much faster than CSO. This is because CSO carries out two processes independently for global (seeking mode) and local (tracing mode) search in each generation which makes it computationally time consuming. However, although GWO has very few parameters to tune and yields improved performance, it takes more time to converge on to the optimum solution as compared to PSO. In PSO, all the particles move through global search and end with local search in the last generation. The momentum effects on particle movement in PSO generally allow faster convergence. On the other hand, in GWO, the adaptive values of parameters $\vec{a}$ and $\vec{A}$ allow a smooth transition between exploration and exploitation. Half of the iterations are devoted to exploration (global search) and the other half are dedicated to exploitation (local search). Furthermore, parameter $\vec{C}$ is used to avoid local optima stagnation, especially in the final iterations. Thus, GWO is more likely to escape locally optimal points and yield a global optimum solution, thus making it more computationally time consuming than PSO as depicted in Table 16.

\section{Conclusion}

This paper has introduced the GWO as an optimization algorithm to the electromagnetics and antenna community. The application of GWO for optimal pattern synthesis of linear antenna arrays was demonstrated for the first time. GWO was applied to obtain the optimized antenna positions and current amplitudes in order to achieve the desired array pattern with minimum SLL along with null placement in specified directions. Suppression of the first side lobe next to the main lobe (near side lobe) while simultaneously controlling the other side lobes was also demonstrated. 
The obtained results have been compared with conventional array (nonoptimized) and with arrays optimized using other nature-inspired evolutionary algorithms such as PSO, ACO, $\mathrm{CSO}$, and $\mathrm{BBO}$. The results suggest that optimization of linear antenna arrays using GWO provides considerable enhancements compared to the uniform array and the synthesis obtained from other optimization techniques. This demonstrates the suitability of GWO to be used by the antenna and electromagnetics community for antenna array optimization.

\section{Competing Interests}

The authors declare that there are no competing interests regarding the publication of this paper.

\section{References}

[1] C. A. Balanis, Antenna Theory and Design, John Wiley \& Sons, New York, NY, USA, 1997.

[2] R. Vescovo, "Null synthesis by phase control for antenna arrays," Electronics Letters, vol. 36, no. 3, pp. 198-199, 2000.

[3] K. Güney and A. Akdagli, "Null steering of linear antenna arrays using a modified tabu search algorithm," Progress in Electromagnetics Research, vol. 33, pp. 167-182, 2001.

[4] M. H. Er, "Linear antenna array pattern synthesis with prescribed broad nulls," IEEE Transactions on Antennas and Propagation, vol. 38, no. 9, pp. 1496-1498, 1990.

[5] M. A. Panduro, D. H. Covarrubias, C. A. Brizuela, and F. R. Marante, "A multi-objective approach in the linear antenna array design," AEU-International Journal of Electronics and Communications, vol. 59, no. 4, pp. 205-212, 2005.

[6] D. Bianchi, S. Genovesi, and A. Monorchio, "Design of linear arrays by employing randomly-overlapped subarrays," in Proceedings of the XXXIth URSI General Assembly and Scientific Symposium (URSI GASS '14), pp. 1-4, IEEE, Beijing, China, August 2014.

[7] Z. Zhang, T. Li, F. Yuan, and L. Yin, "Synthesis of linear antenna array using genetic algorithm to control side lobe level," in Computer Engineering and Networking, pp. 39-46, Springer, 2014.

[8] B. Goswami and D. Mandal, "A genetic algorithm for the level control of nulls and side lobes in linear antenna arrays," Journal of King Saud University-Computer and Information Sciences, vol. 25, no. 2, pp. 117-126, 2013.

[9] V. Murino, A. Trucco, and C. S. Regazzoni, "Synthesis of unequally spaced arrays by simulated annealing," IEEE Transactions on Signal Processing, vol. 44, no. 1, pp. 119-122, 1996.

[10] M. M. Khodier and C. G. Christodoulou, "Linear array geometry synthesis with minimum sidelobe level and null control using particle swarm optimization," IEEE Transactions on Antennas and Propagation, vol. 53, no. 8, pp. 2674-2679, 2005.

[11] Y. Li, F. Yang, J. Ouyang, Z. Nie, and H. Zhou, "Synthesis of nonuniform array antennas using particle swarm optimization," Electromagnetics, vol. 30, no. 3, pp. 237-245, 2010.

[12] N. Jin and Y. Rahmat-Samii, "Advances in particle swarm optimization for antenna designs: real-number, binary, singleobjective and multiobjective implementations," IEEE Transactions on Antennas and Propagation, vol. 55, no. 3 I, pp. 556-567, 2007.
[13] M. Khodier and M. Al-Aqeel, "Linear and circular array optimization: a study using particle swarm intelligence," Progress in Electromagnetics Research B, no. 15, pp. 347-373, 2009.

[14] E. Rajo-lglesias and Ó. Quevedo-Teruel, "Linear array synthesis using an ant-colony-optimization-based algorithm," IEEE Antennas and Propagation Magazine, vol. 49, no. 2, pp. 70-79, 2007.

[15] L. Pappula and D. Ghosh, "Linear antenna array synthesis using cat swarm optimization," AEU-International Journal of Electronics and Communications, vol. 68, no. 6, pp. 540-549, 2014.

[16] S. Pal, A. Basak, S. Das, and A. Abraham, "Linear antenna array synthesis with invasive weed optimization algorithm," in Proceedings of the International Conference on Soft Computing and Pattern Recognition (SoCPaR '09), pp. 161-166, Malacca, Malaysia, December 2009.

[17] S. Karimkashi and A. A. Kishk, "Invasive weed optimization and its features in electromagnetics," IEEE Transactions on Antennas and Propagation, vol. 58, no. 4, pp. 1269-1278, 2010.

[18] W.-C. Weng, F. Yang, and A. Z. Elsherbeni, "Linear antenna array synthesis using Taguchi's method: a novel optimization technique in electromagnetics," IEEE Transactions on Antennas and Propagation, vol. 55, no. 3 I, pp. 723-730, 2007.

[19] U. Singh, H. Kumar, and T. S. Kamal, "Linear array synthesis using biogeography based optimization," Progress in Electromagnetics Research M, vol. 11, pp. 25-36, 2010.

[20] B. Basu and G. K. Mahanti, "Fire fly and artificial bees colony algorithm for synthesis of scanned and broad-side linear array antenna," Progress In Electromagnetics Research B, no. 32, pp. 169-190, 2011.

[21] M. A. Zaman and M. Abdul Matin, "Nonuniformly spaced linear antenna array design using firefly algorithm," International Journal of Microwave Science and Technology, vol. 2012, Article ID 256759, 8 pages, 2012.

[22] M. Khodier, "Optimisation of antenna arrays using the cuckoo search algorithm," IET Microwaves, Antennas \& Propagation, vol. 7, no. 6, pp. 458-464, 2013.

[23] K. Guney, A. Durmus, and S. Basbug, "Antenna array synthesis and failure correction using differential search algorithm," International Journal of Antennas and Propagation, vol. 2014, Article ID 276754, 8 pages, 2014.

[24] K. Guney and A. Durmus, "Pattern nulling of linear antenna arrays using backtracking search optimization algorithm," International Journal of Antennas and Propagation, vol. 2015, Article ID 713080, 10 pages, 2015.

[25] S. Mirjalili, S. M. Mirjalili, and A. Lewis, "Grey wolf optimizer," Advances in Engineering Software, vol. 69, pp. 46-61, 2014.

[26] A. A. M. El-Gaafary, Y. S. Mohamed, A. M. Hemeida, and A.-A. A. Mohamed, "Grey wolf optimization for multi input multi output system," Universal Journal of Communications and Network, vol. 3, no. 1, pp. 1-6, 2015.

[27] L. I. Wong, M. Sulaiman, M. Mohamed, and M. Hong, "Grey Wolf Optimizer for solving economic dispatch problems," in Proceedings of the IEEE International Conference on Power and Energy (PECon '14), pp. 150-154, Kuching, Malaysia, December 20104.

[28] H. M. Song, M. H. Sulaiman, and M. R. Mohamed, "An application of Grey Wolf optimizer for solving combined economic emission dispatch problems," International Review on Modelling and Simulations, vol. 7, no. 5, pp. 838-844, 2014. 
[29] N. Muangkote, K. Sunat, and S. Chiewchanwattana, "An improved grey wolf optimizer for training q-Gaussian Radial Basis Functional-link nets," in Proceedings of the International Computer Science and Engineering Conference (ICSEC '14), pp. 209-214, IEEE, Khon Kaen, Thailand, August 2014.

[30] C. Muro, R. Escobedo, L. Spector, and R. P. Coppinger, "Wolfpack (Canis lupus) hunting strategies emerge from simple rules in computational simulations," Behavioural Processes, vol. 88, no. 3, pp. 192-197, 2011. 


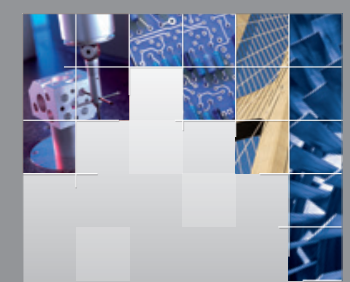

\section{Enfincering}
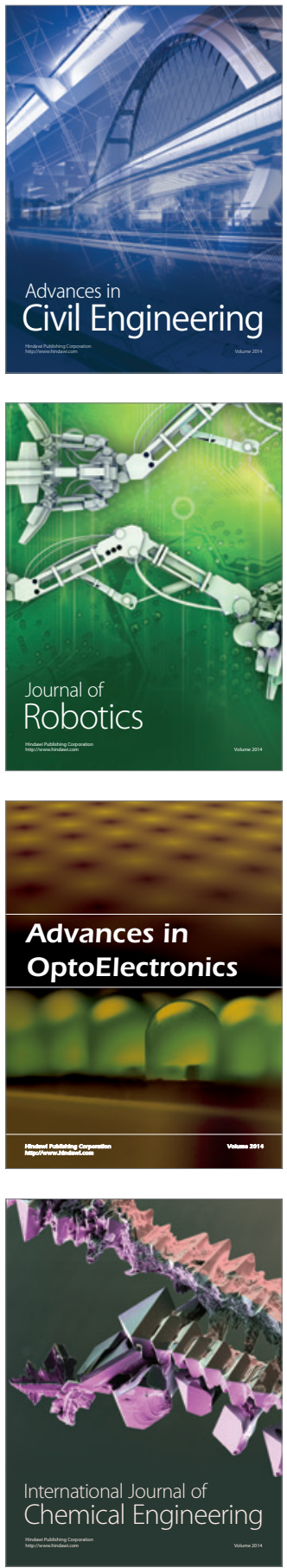

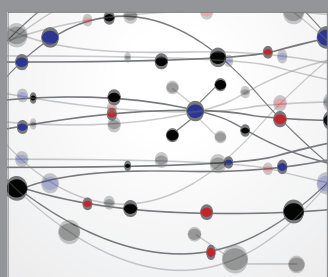

The Scientific World Journal

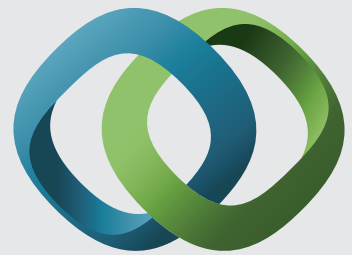

\section{Hindawi}

Submit your manuscripts at

http://www.hindawi.com
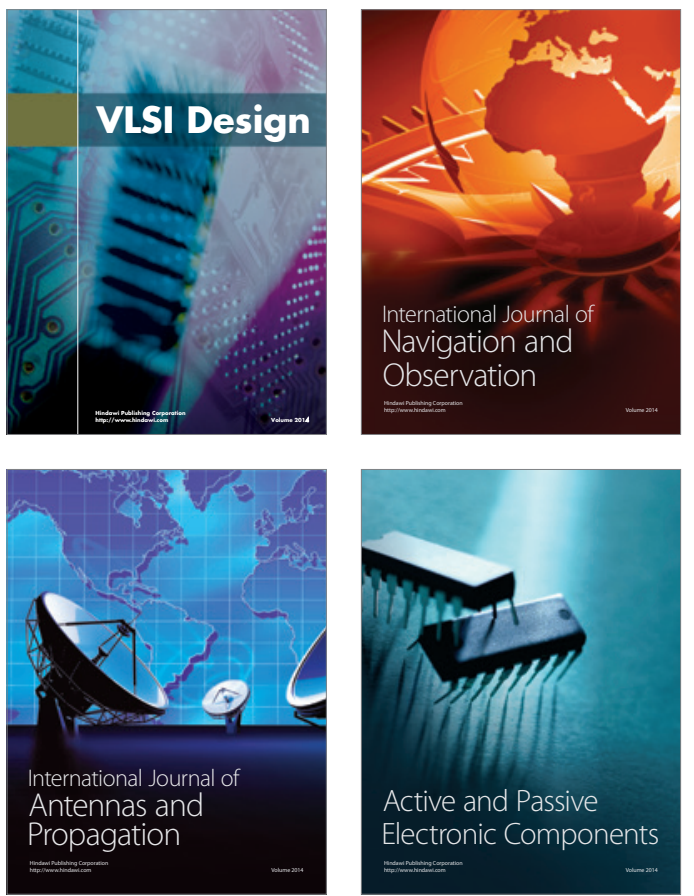
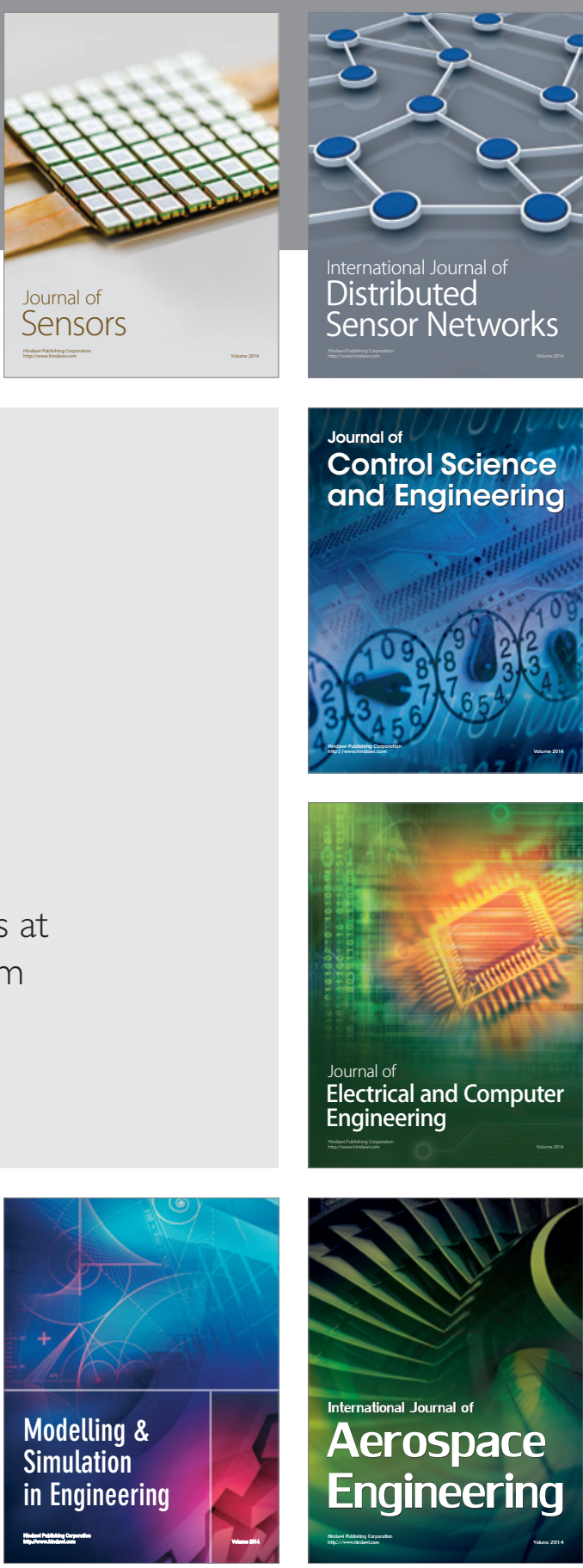

International Journal of

Distributed

Sensor Networks

Journal of

Control Science

and Engineering
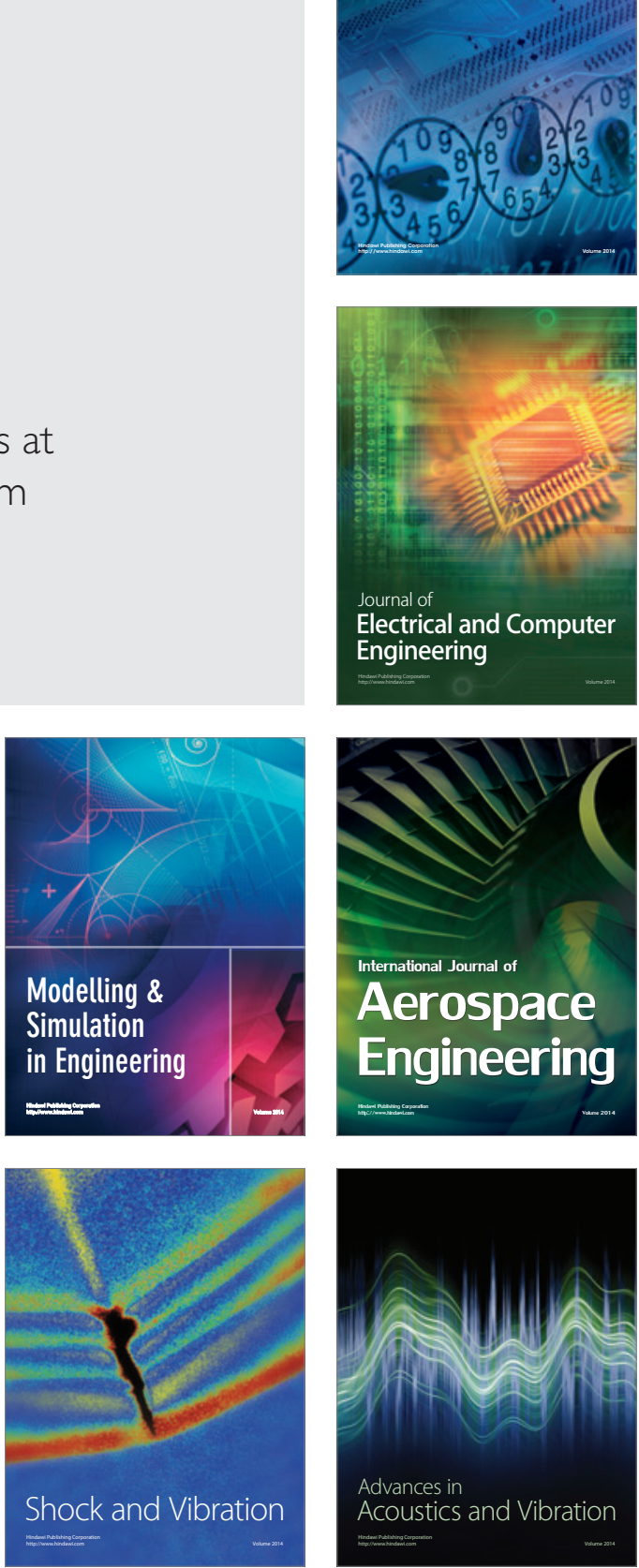